\title{
Enquête
}

Archives de la revue Enquête

5 | 1989

Biographie et cycle de vie

\section{Cycle judiciaire et itinéraires de vie de jeunes délinquants}

\section{Christian Léomant et Nicole Sotteau-Léomant}

\section{(2) OpenEdition}

\section{Journals}

\section{Édition électronique}

URL : http://journals.openedition.org/enquete/89

DOI : 10.4000/enquete.89

ISSN : 1953-809X

Éditeur :

Cercom, Éditions Parenthèses

Édition imprimée

Date de publication : 2 mars 1989

\section{Référence électronique}

Christian Léomant et Nicole Sotteau-Léomant, « Cycle judiciaire et itinéraires de vie de jeunes

délinquants », Enquête [En ligne], 5 | 1989, mis en ligne le 27 juin 2013, consulté le 21 avril 2019. URL :

http://journals.openedition.org/enquete/89; DOI : 10.4000/enquete.89 


\title{
Cycle judiciaire et itinéraires de vie de jeunes délinquants
}

\author{
Christian Léomant et Nicole Sotteau-Léomant
}

1 Pour aménager socialement le temps de vie de chacun, nos sociétés recourent au critère d'âge, pré-organisant de façon "coutumière » ou à l'aide de textes administratifs ou législatifs les rythmes selon lesquels les différentes phases de la vie vont se succéder dans le temps (Langevin, 1987). Cette préstructuration normative du parcours de vie et de ses composantes (familiale, scolaire, professionnelle, etc.) en périodes se succédant participe à la définition des positions de classe et de sexe : elle relève d'abord de la conception d'un temps à structure linéaire, à l'instar du temps-calendrier, aussi bien pour les diverses institutions que pour les acteurs; elle relève aussi d'une conception de l'évolution des capacités humaines (morales, intellectuelles, psychologiques, physiques-productrices et reproductrices) régularisée et normalisée.

2 Une telle organisation de la vie humaine est fondée sur une logique à double face : une logique normative qui concerne les règles et principes devant en particulier régir la vie familiale. S'appliquant de façon différenciée selon les positions sociales, elle est la légitimation de toute intervention et la face visible de la logique de domination dont l'objectif premier est la reproduction des rapports sociaux établis (Boltanski, 1971; Bertaux, 1977 ; Donzelot, 1977 ; Léomant, 1977 ; Commaille, 1981). La question à laquelle nous sommes confrontés est celle de l'impact de tels modèles normalisateurs sur la vie des individus.

3 Dans le champ d'étude de la délinquance juvénile, la perspective théorique dominante y répond en considérant le délinquant comme "une cire molle façonnée par le jeu des structures", une marionnette actionnée d'un monde de systèmes, de logiques, indépendant des individus. Le choix que nous avons fait, dans le cadre d'une recherche sur les processus de production de la délinquance juvénile, de pratiquer une approche biographique en recueillant les récits de vie de jeunes délinquants adultes ${ }^{1}$ est une tentative pour aborder ce champ autrement. Les histoires de vie qui nous furent contées peuvent certes donner l'impression d'être organisées de façon prédéterminée autour de 
faits et d'événements s'enchainnant depuis la naissance et pouvant être classés en différents ordres permettant de reconstituer différentes trajectoires (familiale, scolaire, délinquante, psychiatrique, etc.). Mais les récits eux-mêmes ne se déroulent pas d'une telle manière: ils ne se présentent pas comme des parcours où se cumulent ou se juxtaposent des trajectoires. En effet, l'histoire sociale de ces jeunes doit prendre en compte au moins trois éléments : d'abord les institutions, quelles qu'elles soient, ne sont ni univoques ni autonomes; ensuite toute intervention est médiatisée par un ou des agents particuliers, qui ont leur propre histoire, dans une situation d'interaction de pratiques; enfin le temps vécu par les uns et les autres dans la pratique quotidienne est « fait d'îlots de durée incommensurable, dotés de rythmes particuliers, celui du temps qui passe ou qui piétine, selon ce que l'on en fait, c'est-à-dire selon les fonctions que lui confère l'action qui s'y accomplit » (Bourdieu, 1987) ou selon la qualité de l'événement qui en est l'origine.

4 Ainsi fondée sur la nécessité de saisir une vie dans sa globalité sociale, sa complexité et sa singularité historique, notre démarche nous a conduits à utiliser le concept d'itinéraire social de vie. En effet, s'il est organisé par une position de classe et des contraintes institutionnelles (famille, école, etc.) ou non institutionnelles, un itinéraire social n'est-il pas d'abord le point de rencontre d'un système de relations avec d'autres systèmes de relations (l'un et les autres en évolution continue) ? S'il est le produit de confrontations et d'interactions avec et entre les diverses instances, leurs logiques, leurs temporalités, n'est-il pas aussi l'expression des contradictions entre elles et en leur sein (Léomant, 1987) ? Il nous apparaît alors, du fait de sa complexité et de sa construction interactive, comme le lieu pertinent pour observer comment interfèrent les modèles ayant pour objectif d'aménager le temps social, avec le vécu du temps pratique.

\section{Le cycle judiciaire : un modèle théorique contradictoire en lui-même}

$5 \quad$ Les rapports possibles des justiciables avec les instances judiciaires sont organisés par des textes qui instituent des âges en limites définissant une succession de moments et de phases de vie, et par là même ce que l'on pourrait désigner comme un cycle judiciaire. L'âge de 18 ans, tout particulièrement, apparaît comme la borne différenciant deux statuts, celui de mineur pénal et celui de majeur pénal ${ }^{2}$. Les mineurs pénaux relèvent de la justice des mineurs ${ }^{3}$.

6 La philosophie qui fonde l'ordonnance du 2 février 1945 relative à la délinquance juvénile repose sur la distinction de "nature », pourrait-on dire, entre l'enfant et l'adolescent, d'une part, et l'adulte d'autre part. Elle s'exprime dans un principe qui affleurait déjà

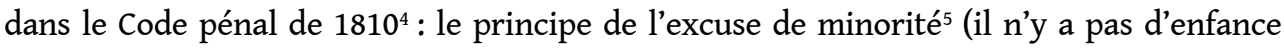
coupable $\left.{ }^{6}\right)$ qui doit réguler les pratiques judiciaires dans le but d'une "rééducation ", d'une " réadaptation », en les orientant vers des mesures éducatives, la répression devant rester l'exception. Pour ce faire, le législateur a organisé cette période de vie de la minorité pénale en plusieurs phases se succédant chronologiquement vers la majorité pénale selon une progressivité de la responsabilité et des peines possibles fondée sur le critère d'âge. Avant 13 ans le principe d'irresponsabilité pénale est absolu ${ }^{7}$. Après 13 ans l'option pénale doit être exceptionnelle ${ }^{8}$. Avant 16 ans la durée de la détention provisoire est limitée à dix jours ${ }^{9}$; après 16 ans aucune limitation de durée. En outre le tribunal pour 
enfants a la possibilité de ne pas retenir l'excuse de minorité et de punir l'accusé comme un majeur.

7 À partir de 18 ans, le majeur pénal dépend des tribunaux pour adultes. Le jugement n'est plus prononcé en fonction de la personnalité et du milieu de l'accusé mais suivant le délit, la carrière criminelle préalable et une échelle des peines où pour tel délit correspond telle peine. C'est alors que non seulement le justiciable devenu adulte perd le droit à l'« éducatif » que lui assurait, en principe, la justice des mineurs et se trouve confronté à une justice essentiellement répressive, mais qu'il va aussi subir la révocation et la mise à exécution des peines avec sursis prononcées lors de sa minorité.

8 La justice des mineurs délègue à la justice des majeurs le soin d'appliquer les mesures répressives qu'elle n'a pas fait exécuter elle-même. Le jeune majeur ne dépend plus d'elle pour les peines qu'elle a prononcées, notamment les peines de prison avec sursis. La justice des majeurs prend en charge, mais uniquement pour l'aspect répressif, la succession de la justice des mineurs. Et c'est ainsi que les tribunaux pour adultes (essentiellement le tribunal correctionnel) vont sanctionner doublement le justiciable : lors du jugement pour un nouveau délit, la condamnation sera cumulée et renforcée par la révocation partielle ou totale des peines avec sursis prononcées lors de la minorité.

9 Les praticiens de la justice des mineurs, notamment au cours de différents travaux consacrés à la protection judiciaire de la jeunesse, ont mis en évidence le «non-sens éducatif » de la rupture des 18 ans. Ils ont insisté sur la nécessité d'une continuité de l'intervention et d'une prise en compte du «phénomène de la délinquance des jeunes adultes » comme l'ont fait certains pays.

10 Que signifie une majorité pénale à 18 ans alors que l'évolution actuelle de nos sociétés, caractérisée démographiquement par une plus grande espérance de vie et le vieillissement, pose le problème des âges d'entrée dans la vie adulte? Les processus sociaux de socialisation et d'insertion montrent un bouleversement du cycle de vie des jeunes «marqué par un report de plus en plus grand du passage du jeune à l'état de producteur au plan économique et de reproducteur au plan démographique " (Commaille, 1986). Le flou, le précaire, l'instable s'installent et « envahissent le champ des modèles temporels où le temps salarié est un enjeu social » (Langevin, 1987). Nombre de travaux et propositions concernant l'insertion professionnelle des jeunes (tel le rapport Schwartz, 1981) ont remis en cause le seuil des 18 ans et utilisent des catégories d'âges diverses à propos des jeunes, notamment celle des 16-24 ans.

11 Le législateur a donc tenté de combler ce que certains nomment un "vide juridique » concernant la catégorie des jeunes adultes ${ }^{10}$ en adjoignant, par la loi du 11 juillet 1975, un article 10bis à l'ordonnance du 2 février 1945. Cet article instaure une mesure éducative applicable aux mineurs délinquants âgés de 16 et 17 ans, d'une durée de cinq années maximum et pouvant se poursuivre jusqu'à 23 ans sous la responsabilité du tribunal pour enfants (la mise sous protection judiciaire) concurremment avec une peine prononcée par la justice des majeurs ${ }^{11}$.

12 De cette brève présentation on peut retirer d'abord que la loi, qui avait été pensée en fonction d'une évolution progressive des capacités humaines jusqu'à la majorité pénale dans le cadre d'un temps linéaire, s'est traduite dans un modèle où cette progressivité est structurée en classes d'âges de telle sorte que la progression s'effectue non pas de façon continue et homogène, mais lors du passage d'une classe d'âges à une autre, par bonds sinon même par ruptures. Le temps ainsi proposé prend la forme d'une discontinuité 
normalisée. Le critère d'âge fait loi ; il impose ses interdits mais offre ses possibilités plus répressives qu'éducatives; il prend la prépondérance sur le principe de progressivité dans la responsabilité pénale qui pourtant l'ordonnait.

Ensuite on constate qu'il n'y a pas seulement succession des phases de la vie, structurant le passage de l'état d'adolescent à celui d'adulte, du statut de mineur pénal à celui de majeur pénal : il y a chevauchement, superposition, indétermination des statuts et des modèles temporels. La majorité pénale des 18 ans apparaît alors comme une fausse rupture, même si elle connaît une sanction institutionnelle du fait du passage du tribunal pour enfants au tribunal correctionnel, passage qui ne peut que marquer, du fait de la signification sociale qui lui est donnée, l'itinéraire social du jeune délinquant.

\section{Intervention judiciaire et temps de l'insertion}

L'analyse des itinéraires sociaux des jeunes délinquants dont nous avons recueilli les récits de vie rend compte des conditions d'opposition entre l'intervention de l'institution judiciaire et les nécessités de l'insertion sociale dont elle se réclame.

Te au long de la minorité pénale, mesures et peines s'ordonnent bien souvent de façon répétitive (liberté surveillée, placement, incarcération, remise à la famille...) comme si la logique de l'institution était bégayante (Léomant \& Sotteau-Léomant, 1987). Ce qui rejoint les observations faites dans d'autres recherches (CFRES, 1978) : au discours fondé sur l'idée que la répression fait suite à la rééducation lorsque tout a été essayé, s'oppose une réalité constituée par un dosage difficilement lisible de non-intervention, de mesures éducatives et de répression carcérale.

L'empreinte de la loi apparaît alors moins dans la progressivité des mesures éducatives orientées par la nécessité de la réinsertion que dans la progression du nombre des incarcérations et dans la progressivité de leur durée en fonction de la structure par classes d'âges imposée par le modèle législatif, ce que confirme B. Fayolle (1981) en écrivant que «l'exception de la répression tend de plus en plus à étouffer la règle de la rééducation ».

Mais c'est la période de passage du statut de mineur à celui de majeur, phase essentielle $\mathrm{du}$ processus de socialisation, qui rend le mieux compte de l'inadéquation de l'intervention de la justice. Examinons quelques faits tirés de l'itinéraire d'un des jeunes.

De 14 à 18 ans, Bruno connaît dans le cadre de la justice des mineurs une alternance de mesures éducatives et de peines d'incarcération dont certaines avec sursis (il en cumule 22 mois). À 18 ans, il est condamné par le tribunal correctionnel à deux ans de prison ferme. Au cours de sa détention, il est condamné pour un autre délit, par un autre tribunal, à huit mois de prison et à la révocation de tous ses sursis. À 20 ans il est mis en liberté conditionnelle. Durant plusieurs mois, il ne commet aucun délit et commence à travailler. Convoqué à la gendarmerie, il est emmené en prison : le parquet a décidé de mettre à exécution à la fois la peine ferme de huit mois et la révocation des sursis.

Première remarque. Pour comprendre ce qui s'était réellement passé et que Bruno ne pouvait expliquer, nous avons dû rencontrer plusieurs acteurs compétents et concernés par le dossier: un éducateur de probation, un juge pour enfants, un avocat, un juge d'application des peines. Ce ne serait qu'anecdotique si ce n'était pas symptomatique d'une institution qui, loin d'être univoque dans son fonctionnement, est un lieu d'obscurités et de juxtapositions qui s'ignorent. Les interventions judiciaires et leurs 
rythmes sont diversifiés selon le lieu (parquet, instruction, tribunal pour enfants, tribunal correctionnel...) et selon les corps professionnels.

19 Le seul espoir de Bruno c'est « son juge » (il s'agit du juge pour enfants auquel il a eu le plus souvent affaire au cours de sa minorité) à qui il vient d'écrire : « Il n'y en a qu'un qui peut m'en sortir, c'est Monsieur B. » nous dit-il. Nous avons rencontré Monsieur B. qui nous a confirmé qu'il ne pouvait rien faire alors qu'il avait prononcé, quelques années avant, ces peines de prison avec sursis :

«Je n'ai pas le moyen juridique, je ne peux pas lutter contre ces mois de sursis qu'on a cassés. C'est l'énorme mécanique, Bruno c'est quelqu'un pris dans un rouage pourvu d'automatismes implacables. »

20 Bruno subissait, comme beaucoup d'autres justiciables, les conséquences d'un fonctionnement parfois peu codifié au niveau du temps d'une partie administrative de la justice : la mise à exécution par le parquet des peines et des révocations de sursis pouvant s'effectuer des semaines ou des mois après le prononcé de la mesure par le tribunal.

Deuxième remarque. L'application de la révocation, qui peut être différée, participe d'un processus de report, dans un temps futur non défini, du «prix à payer " pour un délit commis souvent des mois avant le jugement. Les notions de sursis et de révocation possible de sursis appartiennent à une configuration temporelle en opposition avec la façon des jeunes délinquants de vivre le temps dans le moment et qui s'exprime par la fugue, la violence, le mouvement, le délit; elles ne peuvent ainsi être intégrées à un rapport au monde où l'immédiat semble dominant, et perdant leur sens original de sanction dissuasive, elles constituent une opposition à la "résolution positive» des itinéraires de vie.

Dans l'état d'indétermination où se trouvent les jeunes délinquants, caractérisé en particulier par la précarité de leur situation économique et de leur système relationnel ainsi que par un déphasage sinon même une totale extériorité par rapport aux différents cycles sociaux institués, que ce soit la famille, l'école, la formation professionnelle, l'armée, etc., toute sanction de type carcéral survient bien souvent comme une négation de la période qui a existé depuis le délit, de la situation du moment et de ses conséquences possibles; une réinsertion a pu s'amorcer, un changement de statut a pu avoir lieu : mise en ménage, paternité, entrée dans un emploi.

«J'étais avec une fille, elle était enceinte, elle avait réussi peu à peu à me mettre cette idée-là dans la tête que c'était pas une vie, d'arrêter de faire des conneries. Je travaillais, j'avais une place de chauffeur-livreur, je repartais dans le droit chemin... S'ils me font ça [deux ans de prison à faire], moi je vous dis, dans deux ans je sors, je mets la cagoule, je prends le calibre, je rentre dans n'importe quelle banque.» (Extrait d'un récit.)

23 Cette phase des itinéraires, où se déroule ce passage, aussi bien sur le plan pénal que civil, du statut de mineur à celui d'adulte, ce "pivot mythique du cycle de vie » (Langevin, 1987), est en effet non seulement celle du flou, de l'instabilité, mais aussi des changements de situation, des rencontres et des possibilités, donc des choix de vie.

$\mathrm{Au}$ cours des entretiens, moments de réflexion où s'entrecroisent passés, préoccupations du présent, désirs d'avenirs, les narrateurs insistent sur la nécessité du choix auquel ils ont été ou sont confrontés : d'une part la continuation de pratiques délinquantes et d'un mode de vie festif et ludique, marqué par le luxe et l'argent, qui n'est pas celui auquel leur position sociale leur permet de prétendre, en alternance avec des incarcérations de plus en plus longues, des périodes de liberté de plus en plus courtes, et le risque de « se faire 
descendre "; et d'autre part l'arrêt de la délinquance, le renoncement aux plaisirs immédiats, et l'entrée dans le mode de vie de tout le monde, une "vie tranquille», une « vie de travailleur».

Mais ce choix d'" intégration sociale ", ou plutôt ce souci d'avoir un avenir en continuant d'exister, qui nécessite de travailler "honnêtement» (pour le jeune délinquant, voler "c'est un métier", c'est travailler), est en opposition avec l'identité que le jeune s'est forgé (« la vie c'est d'être voyou, c'est pas d'être travailleur ») et qui est renforcée par ses expériences négatives du monde du travail.

C'est au niveau de la sphère familiale qu'apparaissent, pour la plupart d'entre eux, les conditions nécessaires du choix de vie : il s'agit de la rencontre d'une femme et surtout de la naissance d'un enfant.

«La famille avec la femme et l'enfant c'est merveilleux parce qu'on a constamment près de soi une chaleur humaine... L'enfant c'est je crois ce qu'il y a de plus beau. » (Extrait d'un récit.)

L'expérience de la paternité favorise un bilan de vie et sa réorientation :

«On commence à réfléchir, j'ai toujours rien derrière moi, pas de famille, rien ; j'ai connu le luxe, pas le bonheur en fin de compte et c'est le bonheur qui me manquait [...] Quand on est père on a pas le même raisonnement, on assume des responsabilités. » (Extrait d'un récit.)

Quand on est père, il est insupportable de voir sa femme et son enfant derrière une vitre (au parloir) car "on les fait souffrir » et cet état de prisonnier empêche l'homme d'assurer le rôle de mari et de père (apporter « l'argent nécessaire au foyer » et " assurer l'éducation des enfants »). L'enfant est l'« espoir » et l'avenir d'un père qui, à côté de sa femme, doit prendre soutien sur les perspectives d'avenir qu'il veut pour ses enfants. Il ne veut pas voir son fils "derrière les barreaux", il ne veut pas reproduire sa propre histoire.

La peine de prison intervient donc comme une interruption dans un processus de maturation sociale et psychologique, dans une dynamique composée d'initiatives, de bifurcations, de choix effectués au cours de périodes plus ou moins brèves, dont la constitution cumulative, progressive nécessite du temps. Les itinéraires de ces jeunes délinquants sont ainsi de longs cheminements chaotiques, orientés par le désir d'une insertion sociale perçue plus ou moins consciemment comme un possible hors d'atteinte.

«On parle de recyclage, il y en a pas, il y a aucune solution de secours de toute façon... on m'a jamais fait de cadeaux... on m'a appris que la haine, la haine, et puis c'est tout... on a jamais cherché à comprendre, on m'a remis tout seul ou on m'a enfermé... » (Extrait de récit.)

Quel avenir peut être envisagé dans un monde où la vie est " une farce... une épreuve »? Comment faire pour trouver un travail quand on est sans formation? Comment supporter les relations hiérarchisées et la temporalité du monde du travail ? Comment changer des manières d'être et de faire incorporées depuis si longtemps? Comment ne plus être condamné?

Pourtant, scandés par des événements critiques qui ont en particulier perturbé la période de l'enfance, bouleversé leur déroulement et les relations avec les parents, et qui sont jugés en partie ou totalement responsables de la délinquance, ces itinéraires sont un long moment de "temps vécu », de temps de souffrance, de délinquance et d'incarcération, entre la béatitude d'«avant la chute » et un retour toujours possible à un "nouveau commencement " (Eliade, 1963) ; ils sont aussi la quête d'un enracinement et d'un devenir à travers les générations passées et à venir, et dans l'espace, pour retrouver ce qui a été 
perdu, un refuge, une famille-abri ${ }^{12}$ contre les dangers et les violences du monde extérieur.

Le champ d'étude de la délinquance et de la déviance est rarement unifié. Partant d'une dichotomie administrative (qui touche aussi les équipes de recherche) et judiciaire entre justice des mineurs et justice des majeurs, partant aussi de la spécificité des comportements au cours de l'adolescence, les chercheurs se sont spécialisés.

Les approches longitudinales ont certes permis de déborder ces critères d'âge, mais en restant majoritairement centrées sur des objets isolés « à l'état pur » : les comportements, les jugements, etc. L'approche biographique, approche longitudinale elle aussi, propose un autre paradigme en considérant le déroulement d'une vie dans son unité et sa totalité ; d'abord parce que le récit recueilli est le récit d'une vie remémorée, recréée (l'acteur retrace, objective, analyse, donne sens), tant au niveau du réel que de l'imaginaire ; il est le produit de cette vie dans un moment subjectivement vécu et socialement construit; ensuite parce que l'interaction de pratiques qui se met en place dans les entretiens est aussi une confrontation de savoirs différents qui s'interrogent et se confrontent. Il n'y a plus une trajectoire judiciaire d'un côté, une carrière délinquante de l'autre et une histoire familiale par ailleurs; il n'y a plus d'arrêt d'une histoire à 18 ans parce que les structures législatives l'ont ainsi décidé.

La majorité pénale intervient plus sur le plan de la structure judiciaire comme un élément de la parcellisation de la prise en charge judiciaire que comme une rupture dans la progressivité des interventions. Mais, et c'est ce qui est essentiel, elle est en contradiction avec tout objectif de résolution positive des itinéraires des jeunes délinquants.

Dans une période historique de déstabilisation des processus d'insertion sociale et de remise en cause des diverses catégories d'âges, les itinéraires de vie de jeunes dont la situation sociale est parmi les plus précaires restent confrontés à des pratiques judiciaires marquées par la rigidité des textes qui les ordonnent à contre-temps, de façon ahistorique et a-temporelle, biographiquement parlant.

Autant le flou dans les séquences remet en cause les droits et les devoirs liés aux âges, autant la rigidité des bornes d'âges est une négation des dynamiques processuelles (celle en particulier de l'insertion sociale) fondées sur la durée, durée qui diffère selon les individus, leurs positions dans les rapports sociaux, et selon les qualités attribuées aux périodes où se développent ces dynamiques: de la même manière qu'il faut du temps au législateur pour adapter ses textes aux conditions historiques (il a fallu 102 ans pour changer la majorité pénale de 16 à 18 ans, et 183 ans pour faire évoluer la majorité civile de 21 à 18 ans), de la même manière il faut du temps pour que les jeunes puissent constituer l'ensemble des ressources (relationnelles, personnelles, économiques...) qui leur permettront de changer de voie (toute proportion de temps gardée, naturellement !). 


\section{BIBLIOGRAPHIE}

BERTAUX, D. - Destins personnels et structure de classe, Paris, Presses universitaires de France, 1977

(«Politiques »).

BolTANSKI, L. - « Les usages sociaux du corps », Annales des Sciences sociales, 1971, p. 205-253.

BouRdieu, P. - Le sens pratique, Paris, Minuit, 1980 (« Le sens commun »).

CENTRE De Formation ET DE RECHERCHE De L'ÉDuCATION SURVEILlÉE. - Les contrôles des déviances

juvéniles. Processus et parcours institutionnels, Rapport provisoire sur la recherche poursuivie dans le cadre de l'Action programme de la DGRST, Contrôle social de la déviance, Vaucresson, CFRES, 1978, mimeo.

Commaille, J. - Familles sans justice? Le droit et la justice face aux transformations de la famille, Paris, Le Centurion, 1981 (" Justice humaine »). - « Conférence introductive », in Problèmes de jeunesses et régulations sociales, Actes des cinquièmes journées internationales de Vaucresson (mai 1985), Centre de recherche interdisciplinaire de Vaucresson, 1986, vol. 1.

DonZeLot, J. - La police des familles, Paris, Minuit, 1977 ( Critique »).

EliADE, M. - Aspects du mythe, Paris, Gallimard, 1963 (« Idées »).

FAyolle, B. - « Essai d'approche des mineurs multirécidivistes », Annales de Vaucresson, 18, 1981, p. 116-172.

LANGEVIN, A. - « Rythmes sociaux et réinterprétation individuelle des critères d'âge dans le parcours de vie ", Annales de Vaucresson, 26, 1987, p. 169-179.

LÉOMANT, C. - Pratique judiciaire et rapports sociaux, thèse de $3^{\mathrm{e}}$ cycle, 1977, mimeo.

- «Présentation », Histoires de vies, histoires de familles, trajectoires sociales, $\mathrm{n}^{\circ} \mathrm{sp}$. de : Annales de Vaucresson, 26, 1987.

LÉOMANT, C., SOTTEAU-LÉOMANT, N. - « Itinéraires de vie et trajectoires institutionnelles de jeunes délinquants », Annales de Vaucresson, 26, 1987a, p. 199-222. - « Contribution à une sociologie compréhensive de la délinquance juvénile ", in Changement de société et délinquance juvénile. Actes des Sixièmes Journées d'études internationales de criminologie juvénile, Louvain, Acco, 1987b, p. 321-328.

Peyre, V., BAilleAu, F. - «Un aspect du contrôle des déviances juvéniles. Questions à propos des trajectoires d'une population d'adolescents délinquants institutionnalisés », Communication pour le $8^{\mathrm{e}}$ Congrès international de criminologie, 1978 , mimeo.

Prigogine, I., Stengers, I. - Entre le temps et l'éternité, Paris, Fayard, 1988.

SCHWARTZ, L. - L'insertion professionnelle et sociale des jeunes. Rapport au Premier ministre, Paris, Documentation française, 1981. 


\section{NOTES}

1. Les jeunes adultes (19-24 ans) de sexe masculin, dont nous avons recueilli les récits de vie (au cours de plusieurs entretiens avec chacun), pour certains en prison, avaient tous été placés à la suite de délits et avant leur majorité dans un centre d'observation fermé de la région parisienne appartenant à l'Éducation surveillée (ministère de la Justice). Ceux qui furent placés dans ce centre n'étaient pas représentatifs de la délinquance officielle "tout venant »; ils étaient au contraire considérés, dans leur majorité, comme des «mineurs difficiles » tant par le nombre de leurs délits que par les « échecs » des interventions éducatives et judiciaires.

2. La majorité pénale fixée à 16 ans en 1810 est à 18 ans depuis la loi du 12 avril 1906.

3. La justice des mineurs a la particularité d'être définie par deux textes fondamentaux: l'un, relevant du Code pénal (ordonnance du 2 février 1945), s'applique aux mineurs qui ont enfreint la loi pénale; l'autre, relevant du Code civil (loi du 4 juin 1970), protège les mineurs dits «en danger ».

4. L'article $66 \mathrm{du}$ Code pénal de 1810 distinguait les moins de 16 ans ayant agi "sans discernement».

5. « Désormais tous les mineurs jusqu'à l'âge de 18 ans auxquels est imputée une infraction à la loi pénale ne seront déférés qu'aux juridictions pour enfants. Ils ne pourront faire l'objet que de mesures de protection, d'éducation ou de réforme, en vertu d'un régime d'irresponsabilité pénale qui n'est susceptible de dérogation qu'à titre exceptionnel et par décision motivée.» (Exposé des motifs de l'ordonnance du 2 février 1945.)

6. «Il n'y a pas d'enfance coupable, mais des enfants et des adolescents victimes de leur famille, de leur milieu ou de l'hérédité, à protéger, à rééduquer et à réadapter à la vie sociale ; [en conséquence] les notions de délit et de peine sur lesquelles repose le droit pénal, ne doivent pas s'appliquer aux mineurs. » (Rapport annuel de la direction de l'Éducation surveillée, 1947.)

7. Les moins de 13 ans ne peuvent être condamnés à une peine de détention ferme. Le juge d'instruction a la possibilité "par ordonnance motivée et s'il y a prévention de crime», de prendre à leur égard des mesures de détention provisoire «pour une durée n'excédant pas dix jours, aux fins de recherche d'un placement ».

8. En tout état de cause, l'excuse de minorité doit avoir pour effet de réduire la peine légalement encourue de moitié. En outre les mineurs de plus de 13 ans ne pourront être placés dans une maison d'arrêt « que si cette mesure paraît indispensable ou encore s'il est impossible de prendre une autre disposition ».

9. La loi du 30 décembre 1987 relative au placement en détention ou sous contrôle judiciaire énonce le principe qu'il ne peut y avoir «de placement en détention provisoire en matière correctionnelle du mineur âgé de moins de 16 ans » (mesure applicable au 1.3.1989).

10. Le passage de la majorité civile à 18 ans par la loi du 5 juillet 1974 a contribué à poser au niveau judiciaire le problème des « jeunes adultes".

11. Dix ans après la promulgation de l'article 16bis, sur 46000 mesures éducatives et pénales prononcées à l'égard des jeunes délinquants de 16 et 17 ans en 1985, 145 seulement sont des mesures de mise sous protection judiciaire, dont 136 pour des durées d'un à trois ans.

12. Ainsi Georges, dans un long monologue, nous dit, lors du récit de l'enterrement de sa mère, enfant de l'Assistance publique recueilli par une "vraie Nanterrienne » qu'il adorait et que son père refusait de recevoir, comment disparaît la possibilité de son installation sur une parcelle de terre, de son enracinement à sa mère, à lui, à ses enfants possibles, conditions d'une lignée et d'un avenir « normalisé » hors de la délinquance. 